BASIC RESEARCH

\title{
Methotrexate loaded SAE coated coronary stents reduce neointimal hyperplasia in a porcine coronary model
}

\author{
Y Huang, K Salu, X Liu, S Li, L Wang, E Verbeken, J Bosmans, I De Scheerder
}

Heart 2004;90:195-199. doi: 10.1136/hrt.2002.008169

\begin{abstract}
See end of article for authors' affiliations

Correspondence to: Dr I De Scheerder, Department of Cardiology, University Hospital Gasthuisberg, 49 Herestraat, 3000 Leuven, Belgium; Ivan.De.Scheerder@ skynet.be
\end{abstract}

Accepted 29 May 2003

\begin{abstract}
Objective: To evaluate the effect of stent based methotrexate delivery on neointimal hyperplasia. Methods: Stainless steel coronary stents and biological polymer coated (SAE) stents were randomly implanted in coronary arteries of pigs with a stent to artery ratio of 1.1:1. The pigs were killed after five days (10 stents) or four weeks (20 stents). Second, stainless steel coronary stents were dip coated in a $10 \mathrm{mg} / \mathrm{ml}$ methotrexate-SAE polymer solution, resulting in a total load of $150 \mu \mathrm{g}$ methotrexate/stent. SAE coated stents and methotrexate loaded stents were randomly implanted in porcine coronary arteries with a stent to artery ratio of 1.2:1 and followed up to four weeks.

Results : SAE coated stents and bare stents elicited a similar tissue response at five days. At four weeks, neointimal hyperplasia induced by the coated stents was less pronounced than with the bare stents (1.32 (0.66) $\left.v 1.73(0.93) \mathrm{mm}^{2}, \mathrm{p}>0.05\right)$. In vitro drug release studies showed that $50 \%$ of the methotrexate was released in 24 hours, and all drug was released within four weeks. No impact on vascular smooth muscle cell proliferation or viability was observed in in vitro cell cultures. At four weeks the arteries with methotrexate loaded stents had decreased peristrut inflammation and neointimal hyperplasia (1.22 (0.34) $\left.v 2.25(1.28) \mathrm{mm}^{2}, \mathrm{p}<0.01\right)$.

Conclusions : SAE coating had an excellent biocompatibility with vascular tissue. Stent based delivery of methotrexate in the SAE coating effectively reduced neointimal hyperplasia in a porcine coronary stent model, potentially due to reduced peristrut inflammation.
\end{abstract}

1 n-stent restenosis, caused by neointimal formation, has been a vexatious problem of coronary stenting. Although the molecular mechanism of in-stent restenosis is not completely understood, inflammatory response, smooth muscle cell (SMC) dedifferentiation, migration and proliferation, and extracellular matrix formation within the intima have been suggested to play an important part in the development of neointimal hyperplasia. ${ }^{1}$ Local delivery of anti-inflammatory or antiproliferative agents has been shown to reduce neointimal hyperplasia in animal models and clinical trials. ${ }^{23}$ As the pathogenesis of in-stent restenosis is multifactorial and consists of elaboration of cytokines and growth factors, agents with both antiinflammatory and antiproliferative properties have the potential advantage of suppressing neointimal formation.

Methotrexate, a folate analogue, has been used in the treatment of rheumatoid arthritis, psoriasis, and graft versus host disease after transplantation. It has also been used to treat leukaemia, choriocarcinoma, and head and neck cancer. Methotrexate's immunosuppressive effects is by blocking the tetrahydrofolate dependent steps of cell metabolism. It may also directly inhibit the secretion and production of cytokines from inflammatory cells. Furthermore, as a cytotoxic agent, methotrexate may inhibit proliferation of inflammatory cells and induce apoptosis. ${ }^{4}$

Methotrexate incorporated into a polymer matrix, however, has failed to decrease neointimal hyperplasia in a porcine coronary model. ${ }^{6}$ In the present study, we used a biological polymer to deliver methotrexate to the injured coronary artery wall. The biocompatibility of the polymer and the feasibility and efficacy of stent based methotrexate delivery through this polymer were evaluated in a porcine coronary stenting model.

\section{METHODS}

Stent and stent coating

Stainless steel balloon expandable stents, $16 \mathrm{~mm}$ long, were used for these studies. The bare stents were dip coated in a biological polymer (SAE, Global Medical Systems, Zulte, Belgium) or in a $10 \mathrm{mg} / \mathrm{ml}$ methotrexate-SAE solution, resulting in a total load of $150 \mu \mathrm{g}$ methotrexate/stent.

The stents were sterilised with ethylene oxide before implantation in porcine coronary arteries.

In vitro methotrexate release kinetics

Three methotrexate loaded stents were placed in vials containing $1 \mathrm{ml} 0.9 \% \mathrm{NaCl}$ at $37^{\circ} \mathrm{C}$. Ultraviolet absorbance (Cary 4 E spectrophotometer, Varian Inc, Palo Alto, California, USA) was measured at $222 \mathrm{~nm}$ each day for the first 14 days and after three and four weeks to determine the methotrexate release. One control polymer-only stent underwent the same procedure and the ultraviolet absorbance values were subtracted from the values of the drug eluting stents.

Impact of methotrexate on vascular SMCs in vitro: SMC proliferation and viability

SMCs were isolated from New Zealand White rabbit aorta, and passaged and cultured (50000 cells/well) in six-well plates (Corning, Brussels, Belgium). ${ }^{78}$ Every third day, 0, $10^{-8}, 10^{-7}, 10^{-6}$, and $10^{-5} \mathrm{M}$ methotrexate (Sigma, Bornem, Belgium), dissolved in $\mathrm{NaCl}$, or paclitaxel (Bristol-Myers Squibb, Brussels, Belgium), dissolved in $20 \mu \mathrm{l}$ ethanol, was

Abbreviations: IEL, internal elastic lamina; SMC, smooth muscle cell 
added in combination with medium changes. After seven days, SMC proliferation was determined by either cell counting (Coulter Counter) or protein quantification (BCA Protein Assay Kit, Pierce, Antwerp, Belgium). Before protein quantification in the protein assay, cell viability was determined through neutral red uptake. ${ }^{9}$ A viability index for each concentration was calculated as the percentage of neutral red from the control sample divided by the percentage of total protein from the control.

\section{Stent implantation}

Domestic cross bred pigs of both sexes weighing 20-25 kg were used. They were fed a standard natural grain diet without lipid or cholesterol supplementation throughout the study. All animals were treated and cared for in accordance with the Belgium National Institute of Health guidelines for care and use of laboratory animals.

Surgical procedures and stent implantation in the coronary arteries were performed according to the method described by De Scheerder and colleagues. ${ }^{2}$

\section{Biocompatibility of the coated stents}

To evaluate the biocompatibility of SAE coating, 15 SAE coated stents and 15 bare stents were randomly deployed in the right and left coronary arteries of 15 pigs. Arterial segments were selected to obtain a 1.1:1 stent to artery ratio.

The pigs were killed after five days (10 stents) or four weeks (20 stents).

\section{Methotrexate coated stents}

Seventeen SAE coated stents and 20 SAE coated stents loaded with $150 \mu \mathrm{g}$ methotrexate were randomly deployed in the coronary arteries of pigs. Arterial segments were selected to obtain a 1.2:1 stent to artery ratio. Pigs were killed after four weeks to evaluate the effect of local methotrexate delivery on neointimal hyperplasia.

Tissue processing and histomorphometric analysis At five days or four weeks follow up, the pigs were killed and the stented coronary arteries were perfused with a $10 \%$ formalin solution at $80 \mathrm{~mm} \mathrm{Hg}$. The segments were furthermore fixed in a $10 \%$ formalin solution. Each segment was cut into a proximal, middle, and distal stent segment for histomorphometric analysis. Tissue specimens were embedded in a cold polymerising resin (Technovit 7100, Heraus Kulzer GmbH, Wehrheim, Germany). Sections $5 \mu \mathrm{m}$ thick, were cut with a rotary heavy duty microtome HM 360 (Microm, Walldorf, Germany) equipped with a hard metal knife and stained with haematoxylin and eosin, elastic stain, and a phosphotungstic acid haematoxylin stain. Light microscopic examination was performed blinded to the type of stent used. Injury of the arterial wall caused by stent deployment, peristrut inflammation, and thrombus formation was evaluated for each stent strut and graded as described. ${ }^{10} 11$

The mean score was calculated as the sum of scores for each filament divided by the number of filaments present.

Harvested coronary segments were analysed morphometrically in three predefined slices (proximal, middle, and distal stent parts) by using a computerised morphometry program (Leitz CBA 8000, Leitz, Leipzig, Germany). The average of the proximal, middle, and distal parts of each stented segment was used for statistical comparison. The area of the arterial lumen, the area inside the internal elastic lamina (IEL), and the area inside the external elastic lamina were measured. Furthermore, the area stenosis ( 1 - lumen area/IEL area) and the area of neointimal hyperplasia (IEL area - lumen area) were calculated.

\section{Statistical analysis}

Data are presented as mean (SD). In vitro data (proliferation and viability assay) were evaluated by means of a one way analysis of variance and Dunnett's multiple comparison post hoc test. For comparison of histomorphometric data between groups, a non-paired $t$ test was used. A probability value of $\mathrm{p}<0.05$ was considered to be significant.

\section{RESULTS}

\section{In vitro methotrexate release kinetics}

The drug release curve showed that after 24 hours $50 \%$ of the methotrexate had already been released from the stent. After the first 24 hours, the release was slower and was $87 \%$ at one week. Finally, after four weeks of incubation in $\mathrm{NaCl}$ at $37^{\circ} \mathrm{C}$, release was complete (fig 1 ).

\section{Effect of methotrexate on SMC proliferation and viability}

During SMC culture, methotrexate did not have any effect on cell proliferation, whereas paclitaxel concentration dependently reduced both cell count and protein content. However, neutral red staining showed good SMC survival with methotrexate, with a viability index of $100 \%$ at all concentrations, whereas paclitaxel induced $>50 \%$ cell death at the highest concentration (table 1, fig 2).

\section{In vivo biocompatibility of SAE coated stents}

At five days' follow up, both the bare and the SAE coated stents induced an identical histopathological response. The stent filaments were well aligned with the vascular wall. The media was mildly compressed. Arterial injury induced by stent implantation was not significantly different between groups. A thin fibrin layer covering the stent filaments was observed. A few inflammatory cells trapped within a thrombotic meshwork were observed (fig 3A). No significant difference in inflammatory response and thrombus formation was observed between coated stents and bare stents.

At four weeks' follow up, the neointima of both coated and bare stents was well organised and consisted of extracellular matrix with SMCs (fig 3B, C). A few inflammatory cells were seen adjacent to the stent struts. The mean inflammation score $(1.02(0.08) v 1.11(0.30), \mathrm{p}>0.05)$ and arterial injury score $(0.21(0.21) v 0.28(0.41), \mathrm{p}>0.05)$ of the SAE coated and the bare stents were not significantly different. The mean lumen area $\left(5.65(1.60) v 5.21 \quad(1.35) \mathrm{mm}^{2}\right)$, neointimal hyperplasia $\left(1.32(0.66) v 1.73(0.93) \mathrm{mm}^{2}, \mathrm{p}>0.05\right)$, and area stenosis $(20(11) \% v 26(16) \%, p>0.05)$ were similar in the two groups.

\section{In vivo effect of stent based methotrexate delivery on neointimal formation}

Histopathological examination found that the lumen surfaces of both the SAE coated and the methotrexate loaded stents

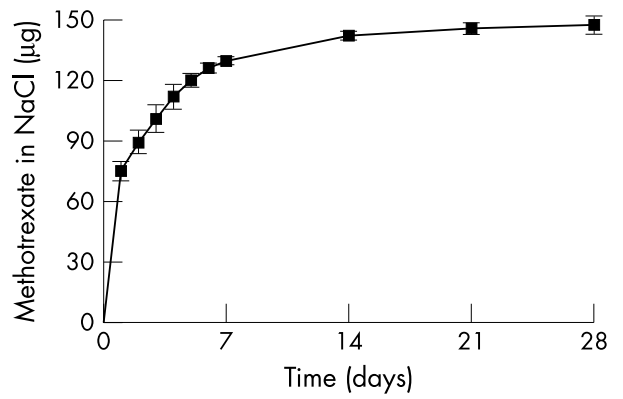

Figure 1 In vitro accumulation of methotrexate released from three SAE coated coronary stents in $1 \mathrm{ml} \mathrm{NaCl}$ at $37^{\circ} \mathrm{C}$. 
Table 1 In vitro assays of rabbit aortic smooth muscle cell proliferation and viability

\begin{tabular}{|c|c|c|c|c|c|c|c|c|}
\hline \multirow[b]{2}{*}{ Drug } & \multirow[b]{2}{*}{ Assay } & \multirow[b]{2}{*}{ No. } & \multicolumn{6}{|c|}{ Concentration } \\
\hline & & & Control & $1 \%$ Ethanol & $10^{-8} \mathrm{~mol} / \mathrm{l}$ & $10^{-7} \mathrm{~mol} / \mathrm{l}$ & $10^{-6} \mathrm{~mol} / \mathrm{I}$ & $10^{-5} \mathrm{~mol} / \mathrm{l}$ \\
\hline \multirow[t]{4}{*}{ Methotrexate } & Cells & 4 & 100 & NA & $108(24)$ & $107(23)$ & $94(18)$ & $100(14)$ \\
\hline & Protein & 3 & 100 & NA & $97(3)$ & $97(6)$ & $95(8)$ & $97(9)$ \\
\hline & Viability & 3 & 100 & NA & $99(6)$ & $99(4)$ & 99 (4) & $98(5)$ \\
\hline & Relative viability & 3 & 100 & NA & $102(6)$ & 101 (3) & $104(10)$ & 102 (5) \\
\hline \multirow[t]{4}{*}{ Paclitaxel } & Cells & 4 & 100 & $100(8)$ & $55(13)^{\star *}$ & $22(7)^{\star *}$ & $13(3)^{* *}$ & $5(2)^{\star *}$ \\
\hline & Protein & 3 & 100 & $97(4)$ & $95(4)$ & $79(3)^{* *}$ & $42(2)^{\star *}$ & $12(9)^{* *}$ \\
\hline & Viability & 3 & 100 & $90(3)^{* *}$ & $88(1)^{* *}$ & $66(4)^{* *}$ & $32(2)^{* *}$ & $5(1)^{* *}$ \\
\hline & Relative viability & 3 & 100 & $94(5)$ & $94(4)$ & $83(7)$ & $76(1)^{*}$ & $46(18)^{\star *}$ \\
\hline
\end{tabular}

Data are mean (SD).

Cells are the number of smooth muscle cells in culture wells as quantified by Coulter Counter; protein is the amount of total protein in culture wells as quantified by $\mathrm{BCA}$ protein assay kit; viability was determined by neutral red staining before total protein quantification; relative viability is the ratio of neutral red staining on total protein quantification. All concentrations are a percentage of the control value.

${ }^{*} p<0.05 ;{ }^{* *} p<0.01$ versus control by one way analysis of variance followed by Dunnett's post hoc test.

$\mathrm{NA}$, not applicable, as methotrexate was dissolved in $\mathrm{NaCl}$.

were covered completely with endothelial cells (table 2). With a greater stent to vessel ratio, medial compression was moderate to severe (fig 3D, E). IEL disruption and medial laceration by stent struts were more frequently observed in the SAE coated stent group. A few sections of the SAE coated stents had a pronounced inflammatory response around some stent struts (fig 3F), although the inflammatory response from the stent struts though the media was rare. The inflammatory response and injury score of the methotrexate loaded stents were significantly lower than those of the SAE coated stents $(1.02(0.03) v 1.27(0.50), \mathrm{p}<0.05$ and $0.20(0.13) \vee 0.41(0.32), \mathrm{p}<0.05$, respectively) (fig $3 \mathrm{G})$, although the mechanical force (oversizing) of the two groups during stent implantation was not significantly different. The ratio of balloon area to IEL area of methotrexate loaded stents was even higher than that of SAE coated stents (1.37 (0.21) v $1.28(0.16), p>0.05)$. Furthermore, the neointimal hyperplasia of methotrexate loaded stents was limited.

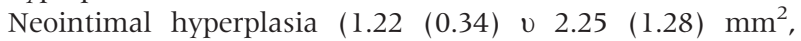
$\mathrm{p}<0.01)$ and area stenosis $(21(8) \%$ v $36(21) \%, \mathrm{p}<0.01)$ of the methotrexate coated stents were significantly lower than those of the SAE coated stents.

\section{DISCUSSION}

In the present study, metal stents coated with a SAE biological coating did not provoke an increased peristrut inflammatory response at five days' and at four weeks' follow up. The neointimal hyperplasia with SAE coated stents at four weeks was even lower than that with bare stents implanted in similar conditions. Impregnation of the SAE coating with $150 \mu \mathrm{g}$ methotrexate significantly reduced peristrut inflammation and neointimal hyperplasia in an overstretched porcine coronary stenting model. This study showed the efficacy of stent based methotrexate delivery in preventing in-stent restenosis.

\section{Biocompatible coating: a crucial determinant for the success of drug eluting stents}

Synthetic polymers have been used as a matrix in which to incorporate drugs for local delivery. With improved coating methods, ultrathin and homogeneous stent coating surfaces can be obtained. However, poor biocompatibility with coronary arterial tissue has been observed with some synthetic polymer coatings. ${ }^{12}$ An inflammatory response to the polymer coating resulting in increased neointimal
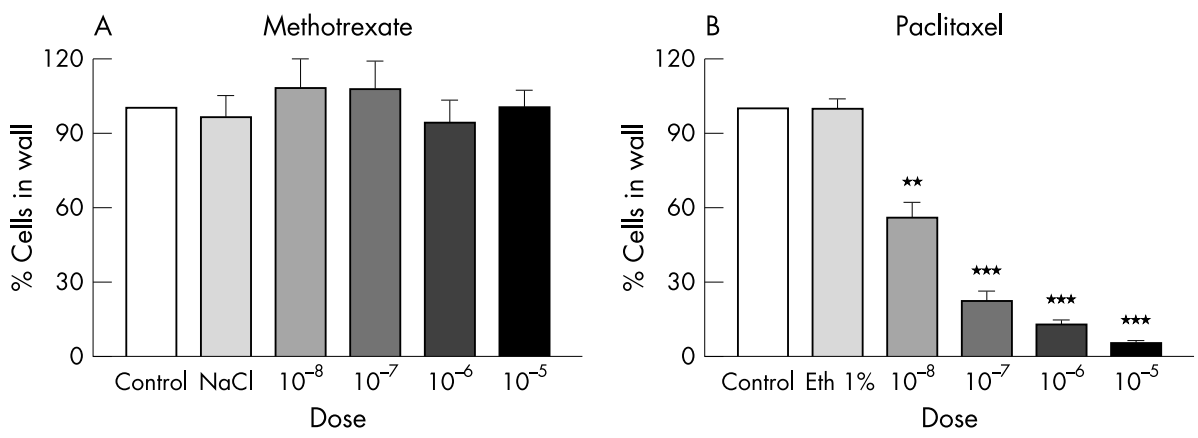

Figure 2 Smooth muscle cell (SMC) proliferation determined by means of (A, B) cell counting and (C, D) total protein assay. SMCs were incubated for seven days with $(A, C)$ methotrexate and $(B, D)$ paclitaxel. ${ }^{* *} p<0.01$; ${ }^{* * *} p<0.0005$ by one way analysis of variance.
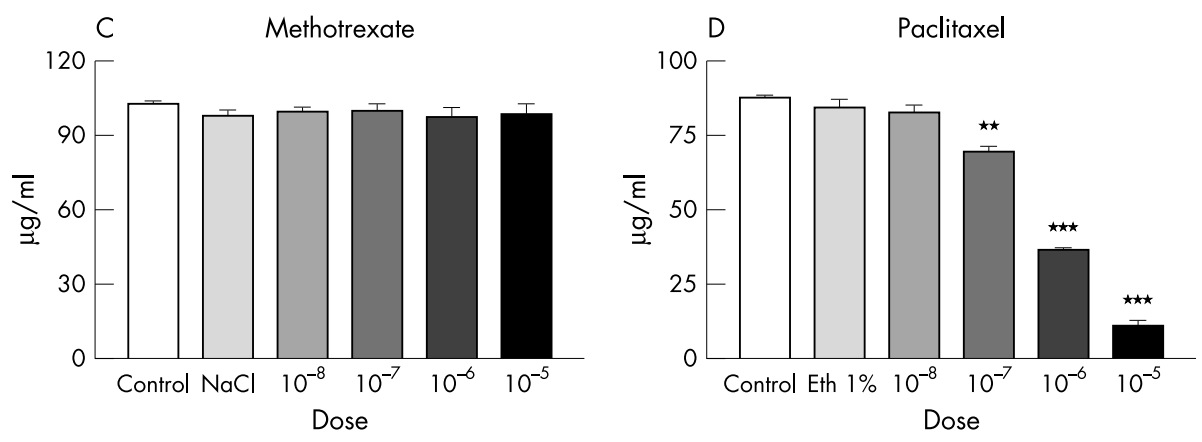

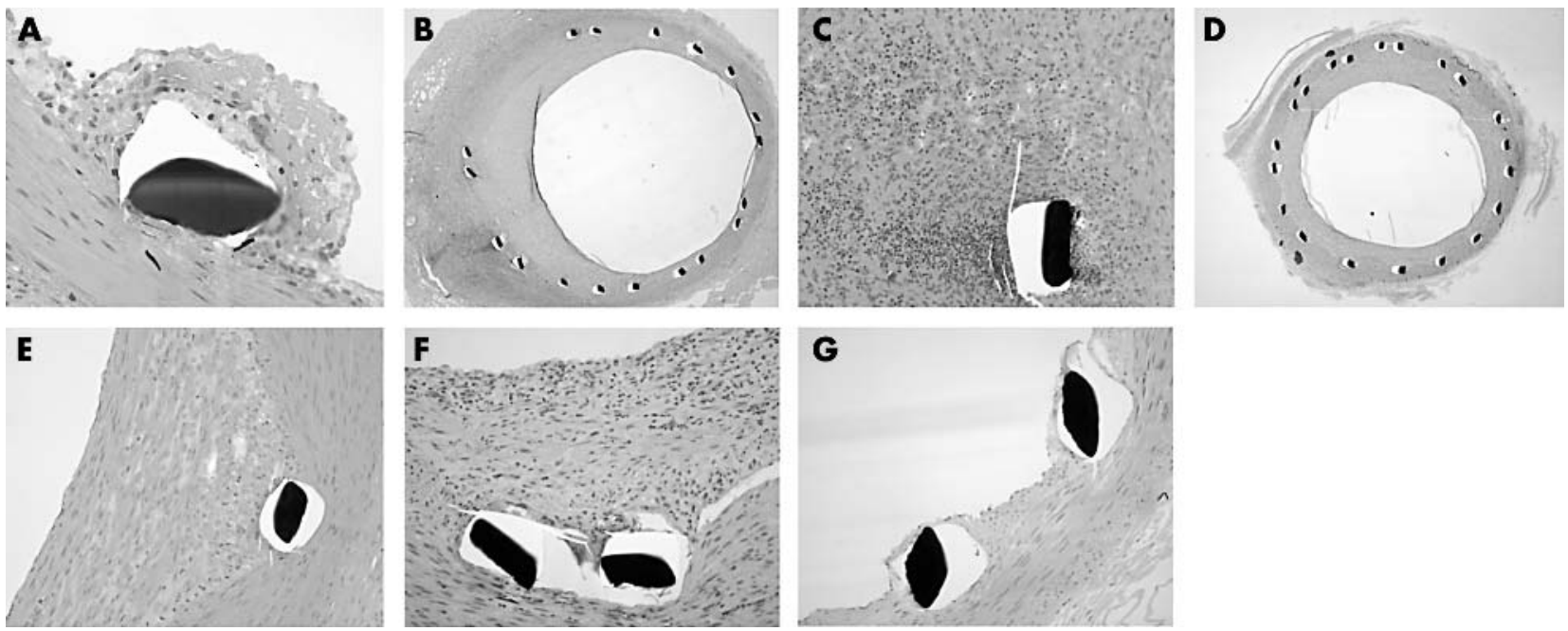

Figure 3 Photomicrograph of a coronary segment stented with a SAE coated stent (A) at five days. A few inflammatory cells infiltrated the thrombus meshwork, with leucocytes rolling on the neointimal surface. Coronary segment stented with $(B, C)$ a bare stent, $(D, E, F)$ an $S A E$ coated stent, or $(G)$ a stent loaded with methotrexate at four weeks. $(B, C)$ An eccentric and well organised neointima is observed. In some stent struts a moderate inflammatory response is present. (D, E) With the increased stent to artery ratio, the media is compressed or even lacerated in some stent filaments. However, only occasional inflammatory cells are observed around the stent filaments. (F) In another case, increased peristrut inflammatory reaction and neointimal hyperplasia are noted. (G) Locally released methotrexate significantly inhibits peristrut inflammation, and neointimal hyperplasia is minimal. $(B, D)$ Haematoxylin and eosin stain. Original magnifications (A) $400 \times,(B, D) 25 \times$, and $(C, E, F, G) 200 \times$.

formation has been reported. ${ }^{12}{ }^{13}$ The increased inflammation may counteract the efficacy of drug eluting stents. In the present study, a biological polymer was applied to a metal stent surface to serve as a matrix for local drug delivery. At five days the histopathological response was the same to the SAE coated stents and the non-coated stents. This low inflammatory response to SAE coated stents was also observed at four weeks' follow up. The neointimal hyperplasia and area stenosis were even lower with SAE coated stents than with the bare stents. All these data suggest that the SAE coating is biocompatible with both blood and coronary arterial tissue. SAE coated stents can therefore serve as a vehicle for local drug delivery.

\section{Methotrexate eluting stents: antirestenotic effects and postulated mechanisms}

It has been found that inflammatory cells and proinflammatory cytokines play an important part in vascular healing. The number of monocytes adhering to the luminal surface of stented arteries correlated linearly with the degree of neointimal hyperplasia. ${ }^{14}$ In transgenic mice, Rectenwald and colleagues ${ }^{15}$ showed that tumour necrosis factor $\alpha$ and interleukin (IL) 1 directly participated in the pathogenesis of neointimal hyperplasia. In addition, the anti-inflammatory cytokine rhuIL 10 reduced intimal hyperplasia after both balloon angioplasty and stent implantation in carotid arteries of hypercholesterolaemic rabbits. ${ }^{16}$

Methotrexate has a variety of anti-inflammatory effects. By inhibition of dihydrofolate reductase and other folate dependent enzymes, methotrexate interferes with DNA synthesis in dividing cells and enhances extracellular adenosine release. Extracellular adenosine, binding to adenosine surface receptors, exerts immunosuppressive effects by inhibiting phagocytosis and producing such proinflammatory cytokines as tumour necrosis factor, IL 2, and IL 12. In addition, methotrexate interferes directly with the cellular response to IL 1 and increases the gene expression of antiinflammatory cytokines IL 4 and IL 10 from stimulated peripheral blood mononuclear cells of patients with rheumatoid arthritis. ${ }^{45}$ Furthermore, methotrexate induces apoptosis of activated $\mathrm{T}$ cells and inhibits recruitment of inflammatory monocytes into inflammatory sites. ${ }^{5}$

These characteristics have led to some enthusiasm for the use of methotrexate to prevent neointimal formation after angioplasty. As oral or intramuscular administration has failed to inhibit neointimal thickening in a porcine coronary stenting model, ${ }^{17}$ intramural balloon catheter delivery and stent based delivery have been explored. Using a perfusion catheter, Muller and colleagues ${ }^{18}$ found that the mean intimal thickness of the methotrexate treated group was not significantly different from the saline treated control group. With cellulose ester polymer coated stents, Cox and colleagues $^{6}$ showed that local delivery of methotrexate did not inhibit neointimal proliferation in stented porcine coronary arteries. Increased arterial injury by the perforated balloon, lack of biocompatibility of the polymer coating, too fast drug release, and inadequate tissue concentrations may all have contributed to the failure of these studies. In this study, we found that the release of methotrexate from the SAE coated stents was slow. In the first 24 hours, $50 \%$ of the methotrexate was released, and release was complete within four weeks. In the overstretched porcine coronary stenting

Table 2 Histophotometric analysis of methotrexate loaded stents at 4 weeks follow up

\begin{tabular}{|c|c|c|c|c|c|c|c|}
\hline Stent coating & No. & $\begin{array}{l}\text { Lumen area } \\
\left(\mathrm{mm}^{2}\right)\end{array}$ & $\begin{array}{l}\text { Neointimal } \\
\text { hyperplasia }\left(\mathrm{mm}^{2}\right)\end{array}$ & $\begin{array}{l}\text { Area } \\
\text { stenosis (\%) }\end{array}$ & $\begin{array}{l}\text { Balloon area to } \\
\text { IEL area ratio }\end{array}$ & $\begin{array}{l}\text { Inflammation } \\
\text { score }\end{array}$ & Injury score \\
\hline SAE & 17 & $4.34(1.85)$ & $2.25(1.28)$ & $36(21)$ & $1.28(0.16)$ & $1.27(0.50)$ & $0.41(0.32)$ \\
\hline Methotrexate & 20 & $5.02(1.42)$ & $1.22(0.34)^{\star *}$ & $21(8)^{* *}$ & $1.37(0.21)$ & $1.02(0.03)^{*}$ & $0.20(0.13)^{*}$ \\
\hline
\end{tabular}


model, locally released methotrexate significantly inhibited peristrut inflammation. In-stent neointimal hyperplasia and area stenosis of methotrexate loaded stents were also significantly reduced. Improved biocompatibility of the polymer matrix with a relatively slow drug release pattern may have accounted for the efficacy of the SAE coated methotrexate loaded stents found in this study.

It has been reported that methotrexate significantly inhibited vascular endothelial cell proliferation in an in vitro cell culture. ${ }^{19}$ Retarded endothelial regeneration after stenting can result in late vascular thrombosis. In our study, methotrexate had no negative effect on endothelial cell regeneration. The lumen surface of methotrexate loaded stents was completely covered by endothelium at four weeks' follow up, and no significant difference was noted between the SAE coated stents and the methotrexate loaded stents.

Vascular toxicity with incomplete healing has been observed with paclitaxel eluting stents. ${ }^{20}$ Methotrexate, as a chemotherapeutic agent, can also influence the metabolism of cells and has a cytotoxic effect. Inhibition of SMC proliferation has been presumed as a potential mechanism in the effect of methotrexate. ${ }^{21}$ In this study, we analysed an in vitro cell culture and compared the effects of methotrexate and paclitaxel on SMCs. The results showed that methotrexate in concentrations from $10^{-8}$ to $10^{-5} \mathrm{~mol} / \mathrm{l}$ had no effect on SMC proliferation and viability. However, paclitaxel was observed to have a dose dependent effect. Therefore, it is not unreasonable to assume that methotrexate is safer and has a wider treatment window than paclitaxel for local treatment. Anti-inflammation, and not antiproliferation, seems to be the main mechanism by which neointimal hyperplasia is decreased.

In summary, the SAE coating was biocompatible with porcine coronary arteries. No retarded endothelial regeneration and other local vascular toxicity were observed. SAE coated stents loaded with methotrexate may eliminate peristrut inflammation, which contributes to reduced neointimal hyperplasia.

\section{Authors' affiliations}

Y Huang, X Liu, S Li, L Wang, E Verbeken, I De Scheerder, Department of Cardiology, Department of Pathology, University Hospitals, Leuven, Belgium

K Salu, J Bosmans, Department of Cardiology, University of Antwerp, Antwerp, Belgium

\section{REFERENCES}

1 Edelman ER, Rogers C. Pathobiologic responses to stenting. Am J Cardiol 1998:81:4E-6E.

2 De Scheerder I, Wang K, Wilczek K, et al. Local methylprednisolone inhibition of foreign body response to coated intracoronary stents. Coron Artery Dis 1996:7:161-6.

3 Sousa JE, Costa MA, Abizaid A, et al. Lack of neointimal proliferation after implantation of sirolimus-coated stents in human coronary arteries: a quantitative coronary angiography and three-dimensional intravascular ultrasound study. Circulation 2001;103:192-5.

4 Hu SK, Mitcho YL, Oronsky AL, et al. Studies on the effect of methotrexate on macrophage function. J Rheumatol 1988;15:206-9.

5 Cutolo M, Sulli A, Pizzorni C, et al. Anti-inflammatory mechanisms of methotrexate in rheumatoid arthritis. Ann Rheum Dis 2001;60:729-35.

6 Cox DA, Anderson PG, Roubin GS, et al. effect of local delivery of heparin and methotrexate on neointimal proliferation in stented porcine coronary arteries. Coron Artery Dis 1992;3:237-48.

7 Seye Cl, Gadeau AP, Daret D, et al. Overexpression of P2Y2 purinoceptor in intimal lesions of the rat aorta. Arterioscler Thromb Vasc Biol 1997:17:3602-10.

8 Bruijns RH, Bult H. Effects of local cytochalasin D delivery on smooth muscle cell migration and on collar-induced intimal hyperplasia in the rabbit carotid artery. Br J Pharmacol 2001;134:473-83.

9 Bonin PD, Singh JP, Gammill RB, et al. Inhibition of fibroblast and smooth muscle cell proliferation and migration in vitro by a novel aminochromone U-67154. J Vasc Res 1993;30:108-15.

10 Schwartz RS, Huber KC, Murphy JG, et al. Restenosis and the proportional neointimal response to coronary artery injury: results in a porcine model. J Am Coll Cardiol 1992;19:267-74.

11 Huang Y, Wang L, Verweire I, et al. Optimization of local methylprednisolone delivery to inhibit inflammatory reaction and neointimal hyperplasia of coated coronary stents. J Invasive Cardiol 2002;14:505-13

12 van der Giessen WJ, Lincoff AM, Schwartz RS, et al. Marked inflammatory sequelae to implantation of biodegradable and nonbiodegradable polymers in porcine coronary arteries. Circulation 1996;94:1690-7.

13 De Scheerder IK, Wilczek KL, Verbeken EV, et al. Biocompatibility of polymercoated oversized metallic stents implanted in normal porcine coronary arteries. Atherosclerosis 1995;114:105-14.

14 Rogers C, Edelman ER. Endovascular stent design dictates experimental restenosis and thrombosis. Circulation 1995;91:2995-3001.

15 Rectenwald JE, Moldawer LL, Huber TS, et al. Direct evidence for cytokine involvement in neointimal hyperplasia. Circulation 2000;102:1697-702.

16 Feldman LJ, Aguirre L, Ziol M, et al. Interleukin-10 inhibits intimal hyperplasia after angioplasty or stent implantation in hypercholesterolemic rabbits. Circulation 2000;101:908-16

17 Murphy JG, Schwart RS, Edwards WD, et al. Methotrexate and azathioprine fail to inhibit porcine coronary restenosis [abstract]. Circulation 1990;82(suppl III):III429.

18 Muller DW, Topol EJ, Abrams GD, et al. Intramural methotrexate therapy for the prevention of neointimal thickening after balloon angioplasty. J Am Coll Cardiol 1992;20:460-6.

19 Hirata S, Matsubara T, Saura R, et al. Inhibition of in vitro vascular endothelial cell proliferation and in vivo neovascularization by low-dose methotrexate. Arthritis Rheum 1989;32:1065-73.

20 Farb A, Heller PF, Shroff S, et al. Pathological analysis of local delivery of paclitaxel via a polymer-coated stent. Circulation 2001;104:473-9.

21 Liang GC, Nemickas R, Madayag M. Multiple percutaneous transluminal angioplasties and low dose pulse methotrexate for Takayasu's arteritis. J Rheumatol 1989;16:1370-3.

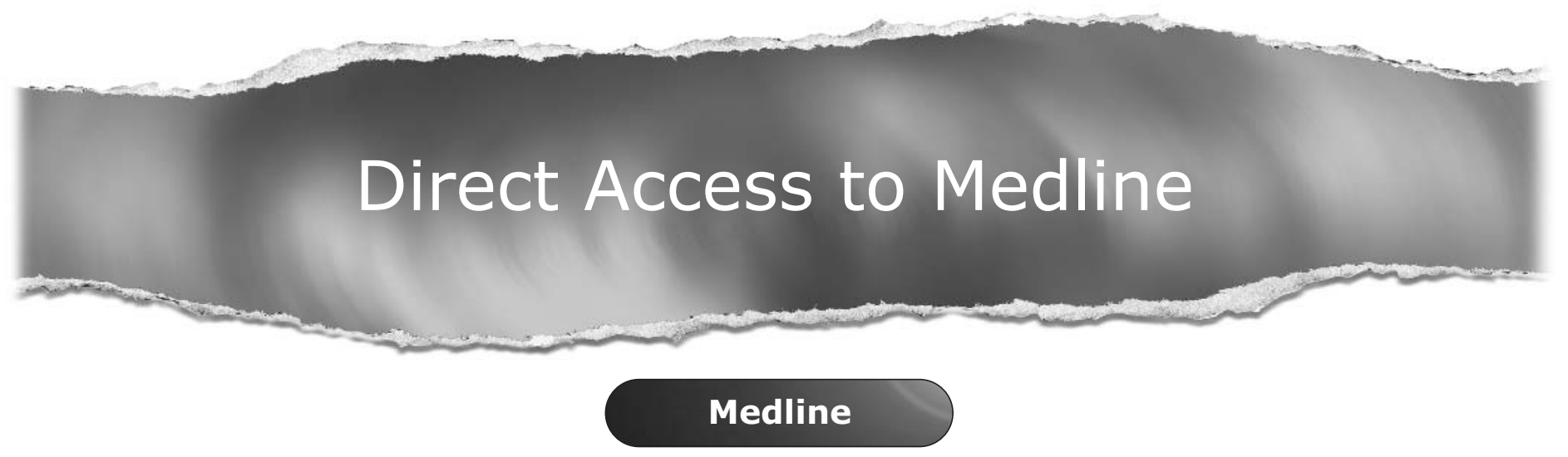

Link to Medline from the homepage and get straight into the National Library of Medicine's premier bibliographic database. Medline allows you to search across 9 million records of bibliographic citations and author abstracts from approximately 3,900 current biomedical journals. 